\title{
STATIC MODULES AND NON-NORMAL CLIFFORD THEORY
}

\author{
J. L. ALPERIN
}

(Received 13 June 1989)

Communicated by B. J. Gardner

Dedicated to G. E. (Tim) Wall, in recognition of his distinguished contribution to mathematics in Australia, on the occasion of his retirement

\begin{abstract}
A version of the Dade-Cline equivalence from Clifford theory is proved for non-normal subgroups of a finite group in the context of a synthesis of a number of equivalences that arise in the representation theory of groups and algebras.
\end{abstract}

1980 Mathematics subject classification (Amer. Math. Soc.) (1985 Revision): primary 20 C 20; secondary 16 A 26,16 A 35,16 A 46.

\section{Introduction}

A recurring theme in representation theory is the connection between a ring and the endomorphism ring of one of its modules: the two rings can be intimately related and one can be used to study the other. Three such results, in particular, are of interest to us: Morita's Theorem, Auslander's equivalence [1; 9, page 429]; and the Dade-Cline equivalence ([3], [2]) from Clifford theory in group representation theory. We shall see that these theorems are themselves all closely related and then use this insight to develop Clifford theory in a new direction. Fix a ring $A$, with unit element; we shall study $A$-modules and we shall always mean left modules. We shall, however, let endomorphisms act on the right; in particular, if $U$ and $V$ are $A$-modules

This research was supported by NSF DMS 8802740 and NSA MDA 904-87-4-2002.

(C) 1990 Australian Mathematical Society 0263-6115/90\$A2.00+0.00 
then, with this action, $\operatorname{Hom}_{A}(U, V)$ is again a (left) module for $\operatorname{End}_{A}(U)$.

The main thrust of Morita's theory is the assertion that if $P$ is a finitely generated projective generator for $\operatorname{Mod}(A)$, the category of $A$-modules, then $\operatorname{Mod}(A)$ and $\operatorname{Mod}\left(\operatorname{End}_{A}(P)\right)$ are equivalent categories and the functor $\operatorname{Hom}_{A}(P,-)$, which sends an $A$-module $U$ to $\operatorname{Hom}_{A}(P, U)$, gives the equivalence. It is also possible to describe the connection between the two rings in terms of tensor products; the use of both the Hom functor and tensor products will be important for us.

Auslander's equivalence is more general and gives a result for any $A$ module, not just projective generators. Let $M$ be an $A$-module; we shall say that another $A$-module $U$ is Auslander (with respect to $M$ ) is there is a presentation (that is, an exact sequence)

$$
\bigoplus^{\prime} M \rightarrow \bigoplus M \rightarrow U \rightarrow 0
$$

such that the application to the functor $\operatorname{Hom}_{A}(M,-)$ gives another exact sequence, namely,

$$
\operatorname{Hom}_{A}\left(M, \oplus^{\prime} M\right) \rightarrow \operatorname{Hom}_{A}(M, \oplus M) \rightarrow \operatorname{Hom}_{A}(M, U) \rightarrow 0 .
$$

Auslander's theorem asserts that $\operatorname{Hom}_{A}(M,-)$ gives an equivalence between the category of Auslander $A$-modules and its image.

The general result of Dade deals with rings that are graded by a group. We shall restrict ourselves to discussing the essential case at the level of group algebras. Let $A=k G$, the group algebra of the finite group $G$ over the field $k$. Let $H$ be a normal subgroup of $G$. Let $N$ be a finitely generated indecomposable $k H$-module which is stable under conjugation by $G$, so, in particular, each $k H$-summand, of the $A$-module $M$ induced by $N$, of the form $g \otimes N$, for $g$ in $G$, is isomorphic with $N$. Let $D$ be the endomorphism ring of $N$ and let $E$ be the endomorphism ring of $M$; so there is a natural embedding of $D$ into $E$. The Dade-Cline result shows that the category of $A$-modules whose restriction to $k H$ is isomorphic with a direct sum of copies of $N$ is equivalent with the category of $E$-modules which are projective as $D$-modules and that this equivalence is given by the functors $\operatorname{Hom}_{A}(M,-)$ and $M \otimes_{E}-$.

We now turn to a synthesis of these results in the next section and then, in the final section, a result about a new kind of Clifford theory.

\section{Static modules}

We now fix an $A$-module $M$ and let $E$ be its endomorphism ring. We shall say that an $A$-module $U$ is static if the natural map (which is the 
evaluation sending $m \otimes \varphi$ to $\varphi(m))$

$$
M \otimes_{E} \operatorname{Hom}_{A}(M, U) \rightarrow U
$$

is an isomorphism. If $V$ is an $E$-module then we say that $V$ is static if the natural map (which, in particular, sends $v$ in $V$ to the map " $m \rightarrow m \otimes v$ ")

$$
V \rightarrow \operatorname{Hom}_{A}\left(M, M \otimes_{E} V\right)
$$

is an isomorphism. The key result that gives the synthesis is the following.

LEMma 1. The category of static A-modules is equivalent with the category of static $E$-modules and the functors $\operatorname{Hom}_{A}(M,-)$ and $M \otimes_{E}-$ give the equivalence.

This has also been discovered by K. Syed [8] who used it to rederive the Dade-Cline equivalence. We shall deduce Auslander's result from it and we shall use it, in the next section, to prove a general theory akin to the DadeCline theorem. The lemma is quite easy to prove directly but it is a special case of a simple and very general result from category theory [4, 5, 6 (page 4), 7] involving any two adjoint functions. The same conclusion holds, though the result is always stated in terms of dualities instead of equivalences; this seems to be the first use of the idea in the case of equivalences. The author here thanks Saunders Mac Lane and Peter T. Johnstone for their helpful assistance with category theory.

In order to derive the Auslander equivalence from the lemma we need only prove the next result.

Lemma 2. The A-module $U$ is static if, and only if, it is Auslander.

Proof. First, suppose that $U$ is Auslander. We have exact sequences for $U$, just as in the definition of Auslander modules above. Tensoring the second with $M$ gives another exact sequence, as tensoring is right exact, so we get a commutative diagram of two exact sequences

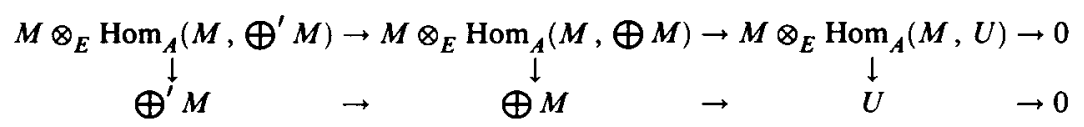

where the vertical maps are the natural ones. Since the first two of these are isomorphisms so is the third, that is, $U$ is static.

On the other hand, suppose that $U$ is static. Since $\operatorname{Hom}_{A}(M, U)$ is an $E$-module there is an exact sequence, a presentation, of the form

$$
\bigoplus^{\prime} E \rightarrow \bigoplus E \rightarrow \operatorname{Hom}_{A}(M, U) \rightarrow 0 \text {. }
$$


If we tensor this with $M$ then we will get a presentation for

$$
M \otimes_{E} \operatorname{Hom}_{A}(M, U),
$$

that is $U$, as follows:

$$
M \otimes_{E}\left(\bigoplus^{\prime} E\right) \rightarrow M \otimes_{E}(\bigoplus E) \rightarrow M \otimes_{E} \operatorname{Hom}_{A}(M, U) \rightarrow 0 .
$$

Applying $\operatorname{Hom}_{A}(M,-)$ we get a commutative diagram

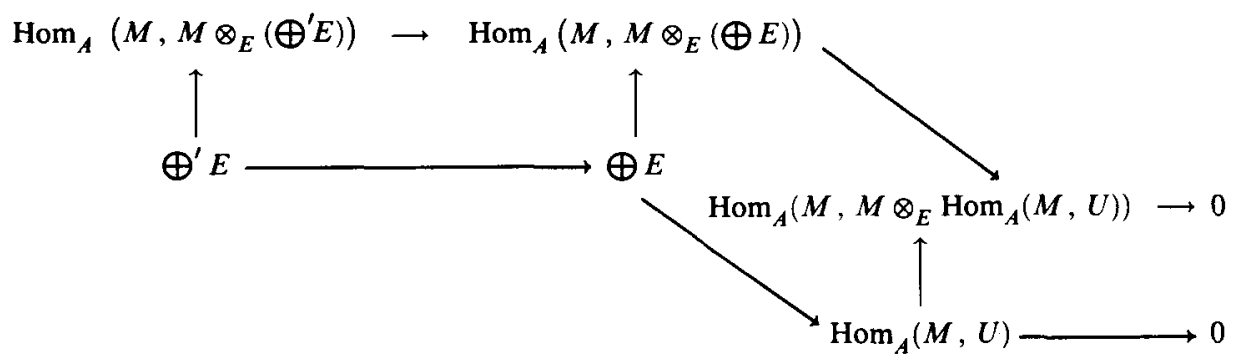

which has the second row exact by definition and the vertical maps all isomorphisms (the third as $\operatorname{Hom}_{A}(M, U)$ is static by Lemma 1 ) so the first row is also exact and $U$ is Auslander.

\section{Non-normal Clifford theory}

We shall now prove a general result about modules induced from subgroups which are not assumed to be normal. We fix a field $k$, a finite group $G$, set $A=k G$, the group algebra, and let $H$ be a subgroup of $G$ and $B=k H$. Let $N$ be a $B$-module and let $M$ be the $A$-module induced from $N$. Set $E=\operatorname{End}_{A}(M)$ and $F=\operatorname{End}_{B}(M)$. We say that the $A$-module $U$ has an $M$-presentation if there is an exact sequence

$$
\bigoplus^{\prime} M \rightarrow \bigoplus M \rightarrow U \rightarrow 0
$$

We say the presentation is $B$-split if it splits upon restriction to $B$. Finally, if $V$ is an $E$-module, then the $F$-module $F \otimes_{E} V$ is called the restriction of $V$ to $F$ (despite the fact that $F$ contains $E$ ) and is written $\operatorname{Res}_{F} V$.

ThEOREM. The category of A-modules with B-split $M$-presentations is equivalent with the category of $E$-modules whose restrictions to $F$ are projective and the equivalence is given by the functors $\operatorname{Hom}_{A}(M,-)$ and $M \otimes_{E}-$.

REMARK. If $N$ and $H$ are as in the introduction ( $N$ is indecomposable, stable and finitely generated while $H$ is normal) then the category of $A$ - 
modules with $B$-split $M$-presentations is easily seen to be the category of $A$-modules whose restrictions to $H$ are direct sums of copies of $N$.

Proof. First, let $U$ be an $A$-module with a presentation as above; we shall show that $U$ is static and that $\operatorname{Res}_{F} \operatorname{Hom}_{A}(M, U)$ is projective. To see that $U$ is static we need only show that $U$ is Auslander, that is, show that the sequence

$$
\operatorname{Hom}_{A}(M, \oplus M) \rightarrow \operatorname{Hom}_{A}(M, U) \rightarrow 0
$$

is exact. But we have a commutative diagram, by Frobenius reciprocity for modules,

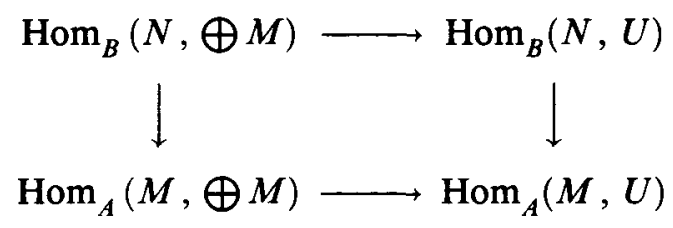

where the vertical maps are isomorphisms. The map in the top row is an epimorphism, as the presentation of $U$ is $B$-split, so the map in the bottom row is also an epimorphism and so $U$ is static. Next, the splitting implies that the $F$-module $\operatorname{Hom}_{B}(M, U)$ is a summand of the free $F$-module $\operatorname{Hom}_{B}(M, \oplus M)$ so that to show $\operatorname{Res}_{F} \operatorname{Hom}_{A}(M, U)$ is projective we need only prove that this restriction is isomorphic with $\operatorname{Hom}_{B}(M, U)$. However, for any $A$-module $W$, there is a natural homomorphism

$$
F \otimes_{E} \operatorname{Hom}_{A}(M, W) \rightarrow \operatorname{Hom}_{B}(M, W)
$$

given by composition: if $f$ is in $F$ and $\varphi$ is in $\operatorname{Hom}_{A}(M, U)$ then $f \otimes \varphi$ is mapped to $f \varphi$ (remember, endomorphisms act on the right). If $W$ is isomorphic with $M$ this map is an isomorphism so the same holds if $W$ is a direct sum of copies of $M$. The argument that proved that $U$ is Auslander showed the sequence

$$
\operatorname{Hom}_{A}\left(M, \oplus^{\prime} M\right) \rightarrow \operatorname{Hom}_{A}(M, \oplus M) \rightarrow \operatorname{Hom}_{A}(M, U) \rightarrow 0
$$

is exact, and we now have a commutative diagram

$$
\begin{aligned}
F \otimes_{E} \operatorname{Hom}_{A}\left(M, \oplus^{\prime} M\right) & \rightarrow F \otimes_{E} \operatorname{Hom}_{A}(M, \oplus M) \rightarrow F \otimes_{E} \operatorname{Hom}_{A}(M, U) \rightarrow 0 \\
\operatorname{Hom}_{B}(M, \oplus M) & \rightarrow \operatorname{Hom}_{B}(M, \oplus M) \rightarrow \operatorname{Hom}_{B}(M, U) \rightarrow 0
\end{aligned}
$$

where the first row is exact and the first two vertical maps are isomorphisms. Since the second row is also exact, by the fact that the presentation is $B$ split, we now have that the third vertical map is an isomorphism and we have proved the claim on the restriction to $F$.

Conversely, suppose that $V$ is an $E$-module whose restriction to $F$ is projective. We shall prove that $M \otimes_{E} V$ has a $B$-split $M$-presentation and 
that $V$ is static. This will prove the theorem: the equivalence of Lemma 1 , restricted to the appropriate categories, then applies. Choose an exact sequence

$$
\oplus^{\prime} E \rightarrow \bigoplus E \rightarrow V \rightarrow 0
$$

so that

$$
M \otimes_{E}\left(\bigoplus^{\prime} E\right) \rightarrow M \otimes_{E}(\bigoplus E) \rightarrow M \otimes_{E} V \rightarrow 0
$$

is also exact and is an $M$-presentation of $M \otimes_{E} V$. We assert that this is $B$-split. If $X$ is any $E$-module then

$$
M \otimes_{E} X \cong M \otimes_{F} F \otimes_{E} X=M \otimes_{F} \operatorname{Res}_{F} X
$$

so the $M$-presentation is of the form

$$
M \otimes_{F} \operatorname{Res}_{F}\left(\oplus^{\prime} E\right) \rightarrow M \otimes_{F} \operatorname{Res}_{F}(\bigoplus E) \rightarrow M \otimes_{F} V \rightarrow 0
$$

which is the tensor product of $M$ with a sequence of $F$-modules which is split since $\operatorname{Res}_{F} V$ is projective. Hence, the presentation is split as a sequence of $B$-modules as asserted. To see that $V$ is static consider the following commutative diagram.

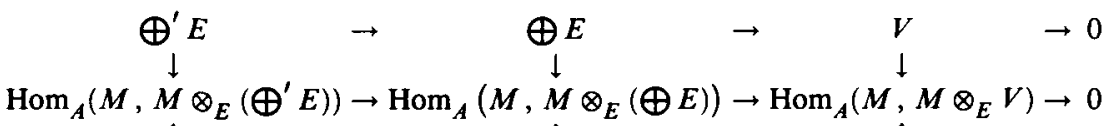

$$
\begin{aligned}
& \operatorname{Hom}_{B}\left(N, M \otimes_{E}\left(\oplus^{\prime} E\right)\right) \rightarrow \operatorname{Hom}_{B}\left(N, \stackrel{\dagger}{\oplus_{E}}(\oplus E)\right) \rightarrow \operatorname{Hom}_{B}\left(N, M \otimes_{E} V\right) \rightarrow 0
\end{aligned}
$$

The first row is exact by definition and the last is exact since the presentation is $B$-split. Moreover, the vertical maps in the first two columns are isomorphisms. Since the vertical map upward in the third column is also an isomorphism, it follows that the second sequence is exact at the last non-zero term, that is, the sequence is exact. Hence, the vertical map downward in the third column is also an isomorphism, that is, $V$ is static.

\section{References}

[1] M. Auslander, 'Representations of Artin algebras', Comm. Algebra 1 (1974), 177-268.

[2] E. Cline, 'Stable Clifford theory', J. Algebra 22 (1972), 350-64.

[3] E. Dade, 'Group-graded rings and modules', Math. Zeit. 174 (1980), 241-62.

[4] J. R. Isbell, 'Top and its adjoint relatives', General topology and its relation to modern algebra, (Proc. Kampur Topological Conf.), Academia, Prague, 1968, pp. 143-54.

[5] J. R. Isbell, 'General functorial semantics. I', Amer. J. Math. 94 (1972), 535-96.

[6] J. Lambek and B. A. Rattray, 'A general Stone-Gelfand duality', Trans. Amer. Math. Soc. 248 (1979), 1-35. 
[7] P. T. Johnstone, Stone spaces, Cambridge Univ. Press, 1982.

[8] D. Sibley, private communication.

[9] Sverre O. Smalo, 'The structure of special endomorphism rings over Artin algebras', Illinois J. Math. 22 (1978), 428-42.

\section{University of Chicago}

5734 University Avenue

Chicago, Illinois 60637

U.S.A. 